ISSN: 1130-3743 - e-ISSN: 2386-5660

DOI: http://dx.doi.org/10.14201/teoredu301273295

\title{
TRAYECTORIAS PROFESIONALES COMPROMETIDAS CON LA EDUCACIÓN DEMOCRÁTICA ${ }^{1}$
}

\author{
Professional pathways committed to democratic education
}

\section{Des parcours professionnels engagés vis-à-vis de l'éducation démocratique}

\author{
Teresa GARCía GÓMEZ \\ Universidad de Almería. Facultad de Ciencias de la Educación. Departamento de \\ Educación. Ctra. Sacramento, s/n. La Cañada de San Urbano. 04120 Almería. \\ Correo-e:tgarcia@ual.es
}

Fecha de recepción: enero de 2018

Fecha de aceptación: marzo de 2018

\section{RESUMEN}

Este trabajo reconstruye las trayectorias profesionales de dos maestras de educación primaria comprometidas con otras formas de hacer escuela. El objetivo de esta investigación era conocer sus aportaciones en la construcción y desarrollo de un modelo democrático de educación. Modelo que se sitúa en lo que denominamos alternativas pedagógicas democráticas, y que definimos en primer lugar para poder contextualizar las prácticas educativas de ambas maestras. El estudio se realiza con una metodología cualitativa, mediante la técnica de entrevistas biográficas para realizar dos historias de vida, que tienen en común haber dedicado parte de sus vidas a un proyecto educativo alternativo. Los resultados muestran dos trayectorias que difieren en el punto inicial de vinculación a un proyecto alternativo de educación,

1. Proyecto I+D «Trayectorias profesionales de maestras, directoras e inspectoras en el sistema educativo español: participación democrática e innovación educativa», financiado por la DGICYT. 
uno es la participación en un proyecto democrático la que concientiza, y otro desde la concientización se busca el proyecto que le permita participar de una educación crítica y transformadora.

Palabras clave: Educación Primaria; educación democrática; alternativas pedagógicas democráticas; maestras; historias de vida.

\section{SUMMARY}

This work reconstructs the professional pathways of two primary school teachers committed to other forms of schooling. The objective of the study was to learn about their contributions to the construction and development of a democratic educational model - a model that would rank as a democratic educational alternative, and which we first define in order to contextualize the educational practices of these two teachers. The study used a qualitative methodology based on biographical interviews to construct the life stories of two teachers, both of whom have dedicated part of their lives to alternative educational projects. The findings show two pathways that differ in the way in which each of the teachers became involved in alternative educational projects: the first, after participating in a democratic project that led to awareness, and the second, moved by awareness to seek a project that would allow her to participate in a critical, transforming education.

Key words: Primary teaching; democratic education; democratic educational alternatives; primary school teachers; life stories.

\section{SOMMAIRE}

Le présent travail décrit les parcours professionnels de deux institutrices engagées envers d'autres façons de faire école. C'est une recherche qui vise à connaître leurs contributions en matière de construction et de développement d'un modèle démocratique d'éducation s'inscrivant au sein de ce qu'il est d'usage d'appeler l'éducation démocratique "alternative», et que nous définirons au préalable pour contextualiser les pratiques éducatives de ces deux institutrices. L'étude a recours à une méthodologie qualitative mettant à contribution la technique des entretiens biographiques, afin de retracer leurs deux histoires de vie. Elles ont ceci en commun qu'elles ont toutes deux consacré une partie de leur vie à un projet éducatif non traditionnel. Mais les résultats révèlent deux parcours bien différents quant à leur lien d'ancrage initial à un projet pédagogique alternatif: chez l'une, c'est la participation à un projet démocratique qui lui a fait prendre conscience, alors que chez l'autre, c'est depuis la conscientisation qu'elle s'est mise en quête d'un projet lui permettant de participer à une éducation critique et porteuse de changement.

Mots clés: Enseignement primaire; éducation démocratique; alternatives pédagogiques; institutrices; histoires de vie. 


\section{INTRODUCCIÓN}

Ante un modelo de educación hegemónico, asentado en políticas y prácticas neoliberales, que mercantiliza e instrumentaliza el conocimiento y la enseñanza en su afán de educar para la integración en el mercado laboral capitalista, competitivo, desigual y explotador, y que convierte al sujeto en objeto de dicha educación; se contrapone otro modelo, atento a dar respuesta a la diversidad de niños, niñas y jóvenes, para potenciar el desarrollo integral que favorezca la formación de una ciudadanía crítica y participativa, donde el alumnado es sujeto de la educación.

Con la intención de visibilizar la contribución de las maestras en este otro modelo de educación, reconstruimos en estas páginas, tras explicar qué entendemos por alternativas pedagógicas democráticas, dos trayectorias profesionales comprometidas con otras formas de hacer escuela, donde la innovación educativa ${ }^{2}$ es una constante, pero no suficiente para la construcción de un modelo contrahegemónico de educación, como sus prácticas evidencian. Prácticas que han contribuido a la creación y desarrollo de dicho modelo, dedicando parte de sus vidas a un proyecto educativo alternativo, y que tienen en común hacer de la escuela un espacio donde la "democracia es más que una forma de gobierno; es primeramente un modo de vivir asociado, de experiencia comunicada juntamente» (Dewey, 1998, 82).

2. LAS ALTERNATIVAS PEDAGÓGICAS DEMOCRÁTICAS: UN MODELO CONTRAHEGEMÓNICO DE EDUCACIÓN

Podemos considerar las alternativas pedagógicas democráticas como proyectos educativos democráticos participativos enfrentados al modelo hegemónico de educación, que tratan de articular libertad, igualdad y justicia con una concepción de democracia crítica y participativa, materializándose dichas características en la gestión, en la transmisión y producción de conocimientos, en el desarrollo profesional docente y en las relaciones internas y externas de los centros escolares (García Gómez, 2004, 2011). Las definimos sintéticamente, recogiendo las características de las escuelas democráticas de un programa de máximos, como:

1. Proyectos pensados y puestos en práctica desde la base (profesorado, padres-madres, alumnado).

2. La teoría y la práctica mantienen una relación dialéctica.

3. Están sustentadas en valores democráticos y de justicia social.

4. Toda la comunidad educativa interviene en la toma de decisiones en los aspectos que conciernen al trabajo diario de la escuela y del aula, mediante

2. La transformación de uno o más elementos de los procesos de enseñanza-aprendizaje y de la organización escolar, sin que dicha transformación llegue a constituir un modelo de educación diferente al hegemónico. 
asambleas y procesos de negociación, buscando compaginar los intereses individuales y los colectivos, para lograr acuerdos que favorezcan el bien común.

5. Rechazan la homogeneización del alumnado, respetando los ritmos y el desarrollo individual, considerando las diferencias individuales y la diversidad como una fuente de riqueza y aprendizaje.

6. Todas las personas (docentes, alumnado, madres-padres, otros miembros de la comunidad) poseen conocimientos y habilidades diversas que son compartidas y enseñadas. El aprendizaje no es unidireccional, sino que todos los sujetos aprenden de todos.

7. El profesorado no es un transmisor del conocimiento, sino un facilitador de las condiciones de aprendizaje para que el alumnado esté motivado, interesado en adquirir nuevos saberes. No es un técnico, sino que controla su trabajo y el currículum que se desarrolla, cuestionando y reflexionando permanentemente sobre su práctica para encauzarla y mejorarla, emprendiendo procesos de investigación acción participativa y crítica.

8. Toda la información circula por diferentes vías para que llegue a todos los sectores de la comunidad educativa y puedan implicarse activamente en la toma de decisiones.

9. El criterio de agrupamiento del alumnado no es la edad, sino los intereses, necesidades y deseos, favoreciendo así las relaciones inter- e intrageneracionales, y enriqueciendo los procesos socializadores.

10. Todos los contenidos son importantes, no hay una jerarquización del conocimiento, ni este se adquiere de forma parcializada sino de forma integral (afectivo, intelectual, manual). Se valoran y se reconocen las distintas culturas, no solo la académica, así como sus diferentes formas de expresión.

11. El conocimiento no es algo que tan solo deba ser acumulado, sino que tiene un valor de uso, sirve de base para conocer las relaciones y mecanismos sociales que generan dominación y opresión, así como para conocer la realidad más inmediata y poder actuar sobre ella. Por tanto, el currículum sensibilizaría y concienciaría sobre clasismo, sexismo, racismo, etnocentrismo, la destrucción medioambiental, los sistemas de explotación, etc., que limitan la construcción y el desarrollo de la democracia; estudiando alternativas, que al mismo tiempo formen en una ciudadanía crítica y activa.

12. Se utilizan diversos y variados recursos para acceder a informaciones múltiples y diversas que posteriormente serán contrastadas. No se utiliza con exclusividad ni como fuente principal de información el libro de texto, el cual suele proporcionar una información sesgada y reduccionista, además de reproducir y ayudar a mantener las posiciones dominantes.

13. La evaluación es formativa y continua para reorientar el proceso de enseñanza-aprendizaje y potenciar este, y nunca para jerarquizar, etiquetar, segregar o limitar. Se realizan prácticas de autoevaluación y coevaluación, en la que participan diferentes miembros de la comunidad educativa. 
Las alternativas pedagógicas democráticas son una construcción colectiva, que conforma redes orientadas a crear espacios y relaciones que autogestionen necesidades básicas -expresivas, afectivas y culturales-, a partir del procomún, de aquellos bienes, vínculos y reglas comunes y compartidos.

\section{MÉTODO}

El estudio que en estas páginas se presenta forma parte de una investigación más amplia, desarrollada con una metodología cualitativa, interesada en conocer la contribución de las maestras al campo de la educación, al ser esta una profesión feminizada básicamente en lo que se refiere a los primeros niveles de enseñanza (infantil y primaria). Investigación que tenía como objetivo general: analizar sus diversas contribuciones a la igualdad social, a la participación democrática y a las diversas formas de transformación educativa, tanto teóricas como instrumentales y metodológicas, tomando en cuenta sus propias prácticas profesionales. Y como objetivo específico: conocer la contribución de estas mujeres a la creación y desarrollo de centros y proyectos educativos, de corte igualitario, democrático y crítico, como parte activa de ellos.

Para ello hemos realizado dos historias de vida de caso único (Pujadas, 1992) a dos maestras, para conocer en profundidad sus trayectorias profesionales. Una, Lola Pedrosa, que ejerció durante cuarenta y tres años como maestra, iniciándose en una escuela unitaria segregada en una zona rural de la provincia de Madrid. Posteriormente, fue trasladada a una escuela unitaria mixta rural de Extremadura. Tras cuatro años decidió ejercer como maestra en un centro privado en la provincia de Madrid, que conocía, grosso modo, por ser un centro innovador. Trascurridos cuatro años decidió marcharse de este centro por la ideología del mismo, coincidiendo esta decisión con la posibilidad de iniciar el proyecto educativo del ceIP Trabenco en Leganés (Madrid), en el que participó durante dieciocho años. Tiempo que le llevó a dar por concluida esta experiencia y, después de un año sabático, solicitó su incorporación en un centro público de la localidad de Rivas-Vaciamadrid (Madrid), centro en el que se jubiló pasados unos años. Otra, Consuelo Uceda, con treinta y nueve años de experiencia, de los cuales nueve estuvo desempeñando diferentes puestos en la administración educativa. Comenzó a ejercer como maestra en una escuela privada de Madrid mientras se preparaba las oposiciones. Una vez aprobadas estas, su primer destino fue una escuela segregada rural. Tras esta experiencia decidió seguir estudiando, buscar respuestas a realidades concretas, y terminada la licenciatura de Pedagogía se incorporó a la enseñanza, desempeñando su labor en diferentes centros públicos de Madrid. Tras catorce años de ejercicio docente ininterrumpido desempeña distintos cargos en la administración educativa y una vez que dio por concluida esta etapa se incorporó a un centro público de la sierra de Madrid en el que permaneció durante siete años, ya que decidió trasladarse y participar en el proyecto educativo del CEIP La Navata en Galapagar (Madrid), ejerciendo ocho años como maestra en este hasta su jubilación. 
Ambas maestras fueron seleccionadas para la investigación por su participación en proyectos (CEIP Trabenco y CEIP La Navata) que se enmarcan en lo que consideramos alternativas pedagógicas democráticas, por su valor testimonial en la participación de este tipo de proyectos. Para dicha reconstrucción no solamente hemos atendido a sus relatos de vida, sino que también hemos considerado documentación adicional (escritos de las maestras, proyectos de centro, memorias...) que nos ha permitido la reconstrucción de ambas biografías de forma más exhaustiva y objetiva posible.

Hemos realizado dos entrevistas a cada una de ellas en diferentes encuentros, obteniendo algo más de cuatro horas de grabación con las entrevistas realizadas a Lola Pedrosa y casi ocho horas y media con las llevadas a cabo a Consuelo Uceda, con el objetivo de mostrar su testimonio subjetivo, tanto de los acontecimientos que han vivido como de las valoraciones que hacen de ellos. Dado que lo importante en las entrevistas es conocer y comprender cómo los sujetos explican los distintos acontecimientos y el sentido que otorga a los mismos, nuestro papel como investigadoras ha sido inducir a la narración, transcribir las entrevistas, ordenar la información de los relatos obtenidos en las diferentes sesiones de entrevista e ir indagando para cubrir los huecos informativos olvidados por los sujetos. Todas las preguntas fueron lo más abiertas y generales posibles (formación realizada, reconstrucción de la trayectoria profesional, razones por las que eligen los destinos profesionales, innovaciones en sus prácticas docentes en relación al modelo de enseñanza dominante, sentido de las mismas, identidad profesional en relación al modelo existente para las mujeres de la época y experiencias que las marcan como profesionales), excepto cuando se trataba de solicitar aclaraciones o ampliaciones a cosas ya dichas. En definitiva, se ha tomado como punto de partida los propios relatos para el desarrollo de las entrevistas biográficas. Estas han consistido en un diálogo abierto con pocas pautas, en el que la función básica de la entrevistadora ha sido estimular el habla de las dos maestras entrevistadas para que proporcionaran respuestas claras, cronológicamente precisas, en las que explicitaran de la forma más amplia posible las referencias a terceras personas, a ambientes y lugares concretos en los que transcurrieron sus distintos episodios biográficos.

Cada uno de los relatos biográficos ha sido categorizado y clasificado, es decir, reducido a categorías analíticas para ir realizando el análisis de contenido, que ha consistido en la realización de una descripción objetiva y sistemática de los textos obtenidos, de manera que se ha realizado un trabajo de construcción textual que ha ido hilvanando cronológicamente los discursos de las maestras.

\section{Resultados}

Reconstruimos dos trayectorias profesionales que difieren en el punto inicial de vinculación a un proyecto alternativo de educación, en un caso es la participación la que concientiza, y otro desde la concientización busca el proyecto que le permita participar de una educación crítica y transformadora. 


\subsection{Dos trayectorias profesionales: de la innovación educativa a la alternativa pedagógica democrática}

Lola Pedrosa y Consuelo Uceda estudian Magisterio durante el franquismo por decisión familiar, por la situación económica y especialmente porque, al igual que para otras muchas mujeres, el Magisterio era una buena salida profesional para niñas de 14 años al ser una carrera corta y acorde con el modelo de feminidad.

4.1.1. La concientización a través de la participación en un proyecto de educación alternativo

Lola Pedrosa no ha sido maestra por vocación, sí por decisión y convicción: "Yo no quería ser maestra. [...] Pero luego me gustó, y es un trabajo que no cambiaría por ningún otro» (2. ${ }^{a}$ Entrevista, 52-53). Ha ejercido durante 43 años, y en su trayectoria profesional podemos situar un antes y un después. La línea de dicha división viene marcada por su participación en la gestación y desarrollo del proyecto educativo del ceIP Trabenco ${ }^{3}$ (Leganés-Madrid), por lo que esta supuso para ella, "mi vida entera», personal y profesionalmente, y para una comunidad.

El antes se inicia a la edad de 17 años. Sus primeras experiencias como maestra las tiene en dos escuelas unitarias rurales - una en la provincia de Madrid y otra en la de Extremadura-. En la primera, una escuela segregada, permanece un año. En la segunda, mixta por razones económicas del municipio que imposibilitaba la segregación por sexo y por edad, con un elevado número de alumnado, permanece durante cuatros años, en los que su prioridad era "Sobrevivir" y aprender dada su inexperiencia. Sin un planteamiento crítico de la enseñanza, son las condiciones de trabajo -niños y niñas de distintos niveles educativos y diferentes edades en el aula, número elevado de alumnado- y las condiciones meteorológicas de la zona las que hacen que introduzca aspectos innovadores: currículum unificado para ambos sexos; trabaja con grupos, potenciando la tutorización entre iguales para poder atender a los diferentes niveles en el aula; y mantiene la escuela abierta hasta las diez de la noche, para procurar un lugar caliente para los niños y niñas.

Deja la escuela rural para ir a trabajar con una amiga a un centro privado, del Opus Dei en Madrid, que realiza innovaciones, pero sin saber la dirección de las mismas. Aquí conoce otras realidades y experiencias educativas nacionales e internacionales, y logra introducir algunas innovaciones en su aula: trabajos más prácticos y asambleas de aula. En dicho centro permanece cuatro años, y decide marcharse por el control y las presiones ideológicas.

Durante los últimos años en este centro proporciona información y documentación sobre innovación educativa a un amigo, Julio Delgado ${ }^{4}$, para el colegio que

3. http://www.trabenco.com/.

4. Quien inicia como coordinador los colegios Trabencos (1. ${ }^{\mathrm{a}}$ Entrevista, 14). 
estaba creando la Cooperativa de Viviendas Trabenco (TRABajadores EN COmunidad) en Entrevías (Vallecas-Madrid), para que asistieran los hijos e hijas de los trabajadores y las trabajadoras de dicha cooperativa. Se produce un intercambio, Lola le proporciona ideas pedagógicas a Julio y este a ella ideas políticas, ideas que provocan el inicio de una conciencia política en ella, como más adelante abordaremos.

Él (Julio Delgado) me plantea que por qué no colaboro en pasarle ideas y hay un intercambio curioso porque me pasa cuestiones políticas prohibidas aquí y yo le paso cuestiones pedagógicas y se pone en marcha Trabenco de Entrevías, un año antes que el Trabenco de Leganés, se ponen en marcha asumiendo ideas, de todas estas innovadoras de las que yo ya tenía... Entonces yo no tenía una excesiva conciencia política, empiezo a tomar conciencia política en este intercambio con este amigo y viendo las realidades de allí, y de las gentes que llegan al Trabenco de Leganés. La gente que viene a la cooperativa de Trabenco viene de chabolas, de casas bajas de Vallecas y cuentan sus historias que son muy duras, pues claro, tomas conciencia, yo tenía una cierta conciencia social... (1. ${ }^{a}$ Entrevista, 7-8).

Este mismo proceso ocurre en Leganés un año después, y Julio Delgado le propone a Lola iniciar junto con Federico Martín Nebras el colegio de la Cooperativa Trabenco en Leganés en el curso 1972/1973. Propuesta que coincide con la decisión que había tomado de no continuar en el centro del Opus Dei.

La Cooperativa Trabenco está formada por clase obrera con conciencia de clase y un fuerte compromiso social y político. Sus integrantes son militantes de diferentes partidos y organizaciones de izquierdas, con un sentido y práctica comunitaria que van desarrollando a través de la participación de todas las personas socias (Pumares, 2001). Participación que definirá la gestación y desarrollo del proyecto educativo de Trabenco. Concibe el colegio como un centro cultural, que atiende a las inquietudes y expectativas del barrio y de sus integrantes, así como una comunidad educativa de familias, profesorado y alumnado orientada a conseguir una formación integral y un constante desarrollo profesional de los docentes (Cooperativa Trabenco, 1971 cit. por Pumares, 2001), entendiendo la escuela como elemento de y para la comunidad, como motor de desarrollo comunitario.

Lola Pedrosa y Federico Martín son los impulsores iniciales del colegio público Trabenco de Leganés, que comienza a funcionar en enero de 1973 en los locales de las viviendas de la cooperativa, tras llegar a un acuerdo con el Ministerio de Educación en el que esta cedía los locales a cambio de que pudiera proponer y decidir quiénes conformarían el equipo docente de la lista de interinidades.

El centro comienza a gestarse con el mismo mecanismo de organización y toma de decisiones de la cooperativa: la Asamblea. Esta era convocada a diario o casi a diario a la que asistían familias y docentes, eran intensas las discusiones y extensas en duración, al igual que los claustros donde se discutía más cuestiones pedagógicas, se intercambiaban y reflexionaban lecturas de Freire, Freinet, Marta Mata, de la Escuela Moderna, de las experiencias catalanas y de las Escuela de Verano en Barcelona y de las de Acción Educativa. 
Resultado de estas reuniones es el consenso del proyecto político de educación colectivo de Trabenco, con una idea clara por parte de la cooperativa y una apuesta fuerte por lo público, lo común

... ya se empieza con esa mentalidad de escuela distinta, y... se quería sobre todo una escuela antifascista [...] Se inicia un ideario que pactamos entre todos [...], la idea de los cooperativistas era muy buena, era que sus hijos se tenían que educar de otra manera, tenían que abrir los ojos a la realidad crítica sobre todo (1. ${ }^{\mathrm{a}}$ Entrevista, 8-9).

Proyecto político que se crea y se desarrolla con el ejercicio real de una democracia participativa, en el que se elabora una estructura que permite la cogestión, siendo la Asamblea General el máximo órgano de participación y toma de decisiones consensuadas, relativas tanto al funcionamiento del centro como a aspectos pedagógicos, en la que participan todas las familias, todo el profesorado y alumnado de segunda etapa (Cooperativa de Viviendas Trabenco, 1980). Esta estructura era lenta y costosa en tiempo. Por ello, más tarde, la Asamblea General delega en la Junta aquellos asuntos que no son urgentes ni conflictivos. Esta está constituida por dos padres o madres de cada clase y dos docentes. Al mismo tiempo, se van creando comisiones en función de las necesidades (económica, salidas, pedagógica, material, deportes, etc.) para la gestión cotidiana de la escuela. Existe también la Asamblea de Curso, en la que docentes y familias hablan sobre la marcha de la clase y de otros aspectos relacionados con sus hijos e hijas: alimentación, literatura infantil, televisión, etc. (Pumares, 2001).

En la Asamblea General se consensua el concepto de enseñanza, entendiéndola como un proceso activo -eliminando la idea del alumnado como receptor pasivo de información, por la que el papel docente no es transmisor, sino orientador y guía en la formación de personas críticas-; individualizado para potenciar una formación integral; y personalizado y práctico -que le prepare para la vida, para conocer la realidad en la que vive, no solo para una cualificación profesional (Cooperativa Trabenco, 1971 cit. por Pumares, 2001, 35). También se acuerdan los objetivos de los procesos de enseñanza.

Esta concepción de enseñanza y los objetivos marcados guían los permanentes procesos de investigación acción del profesorado en el trabajo que va desarrollando en las aulas. Se realizan diferentes formas de organizar al alumnado, por edad en su clase correspondiente, pero también se lleva a cabo un trabajo interclases e interciclos.

No se usa con exclusividad un libro de texto, sino que este material es diverso, de distintas editoriales, junto con otros libros que forman parte de la biblioteca de aula. Al mismo tiempo, el medio-el barrio y otros contextos son un recurso educativo, por ello las salidas son frecuentes en Trabenco. Las aulas se trasladan a lugares diversos que implican un trabajo interdisciplinar previo por parte del alumnado, durante y posterior a las salidas del centro. Estas son variadas, oscilan entre un día con una clase hasta una semana con el centro en su totalidad. No solo el centro sale 
al barrio -u otros contextos-, sino que este también entra al centro, ya que en él las "puertas» permanecen abiertas para la realización de múltiples y diversas actividades, donde la participación de las madres y padres es una constante. No solo la comunidad participa en el centro, sino que también este lo hace en la comunidad. Es un proyecto educativo que estaba inserto en lo social, en las luchas del barrio, en el desarrollo comunitario

Hay un movimiento sociopolítico fuerte desde el principio, reivindicando cosas y pensando que la escuela tiene que estar incardinada en la realidad social, ¿no? la escuela está tan físicamente en el entorno, que participamos de todo lo que la gente tenía como inquietud. Si hay que pedir un ambulatorio, pues vamos a pedir un ambulatorio con los padres y los niños, ¿no? Y estas cosas... (2. ${ }^{a}$ Entrevista, 23).

La estructura y la organización del aula potencian la participación, autonomía y responsabilidad del alumnado, formando equipos para el funcionamiento del aula (biblioteca, limpieza, etc.), desempeñando papeles distintos dentro de los mismos, los cuales eran rotativos para potenciar aprendizajes diversos. La asamblea de aula define la estructura y la organización de la misma. Asamblea que se realiza semanalmente, de esta salen las propuestas de trabajo a partir de los intereses del alumnado y se realiza una valoración de la semana. En esta valoración el sistema Freinet: felicito, critico y propongo, ocupa un lugar importante, desarrollando la capacidad de crítica y autocrítica para juzgar el trabajo realizado. Funciona la autoevaluación y coevaluación en asambleas para tal fin, dando como resultado una apreciación global del alumno o alumna en cuestión a partir de informaciones procedentes de él o ella misma, del equipo de trabajo, del maestro o maestra y de las familias que también participan. No hay calificaciones ni notas ni entrega de boletines, si bien las hubo durante un tiempo, se decidió eliminarlas totalmente a principios de los años ochenta (Pumares, 2001, 139), matizando Lola el carácter simbólico y obligatorio de las mismas

... había autoevaluación desde el principio y además los alumnos cuando se autoevalúan son incluso más duros que los profesores, con ellos mismos, para esto la clase siempre estaba en corro y hablaban. Había una nota individual, una de equipo y una del profesor, con esas notas se hacía una apreciación global del alumno... (1. ${ }^{\mathrm{a}}$ Entrevista, 16).

Los contenidos se globalizan o se trabajan interdisciplinarmente por medio de proyectos, donde el alumnado aprende a trabajar en equipo buscando, seleccionando y reelaborando la información, que posteriormente expone a compañeros y compañeras o a la comunidad escolar. Metodología que rompe con la jerarquización de las áreas de conocimiento que integran el currículum, valorizando e impulsando la educación plástica, el texto libre y la literatura (Pumares, 2001). Los saberes y las aficiones también formaban parte de estos al ser compartidos con el grupo-clase: «Él era coleccionista de minerales y entonces hacíamos que diera conferencias de minerales en las clases» (1. ${ }^{a}$ Entrevista, 35). 
Los contenidos se abordan desde un enfoque materialista y con invitados e invitadas - del ámbito de la literatura, música, etc.- comprometidos con la situación social y política del momento. Estos son tratados para potenciar su valor de uso, y no de acumulación para su reproducción.

La coeducación formaba parte desde sus inicios en Trabenco. El primer año comienza con aulas segregadas por sexo, no cubriendo sus objetivos iniciales por los múltiples problemas que tuvo en su apertura el centro (Pumares, 2001). Es en el curso académico siguiente, 1973/1974, cuando todos los cursos son mixtos, y se incorporaron temas y actividades para reivindicar y potenciar la igualdad entre hombres y mujeres, entender la situación de las mujeres y la realidad del alumnado en el ámbito familiar según su sexo.

Fue pionero porque, el primer año no había coeducación 5 y la implantamos sin más, decidimos que chicos y chicas juntos. Esto nos trajo problemas a mansalva con la inspección y luego al poco tiempo ya, se aceptó la coeducación, pero la implantamos antes, y teníamos discusiones enormes [...] Se intentaba que hubiera... en la clase una igualdad, aunque había sus temas, eran niños de una procedencia económica y social muy baja con lo cual el machismo estaba presente incluso en gente de izquierdas muy comprometida, nos sorprendía (1. ${ }^{a}$ Entrevista, 12$)$.

La igualdad entre sexos se asumía como parte de la lucha de clases, formaba parte de la cotidianidad, de la organización del aula y del centro

... no había una conciencia tan clara, pero yo creo que la mayoría de las mujeres que estábamos allí, estábamos por la igualdad y la vivíamos y punto, no nos planteábamos una militancia especial. [...] Pero no era esa conciencia separada de lo demás, no... digamos que la gente defiende que la lucha feminista es una lucha de clases, pues estaba incluida en la lucha de clases, ¿no? Era un movimiento por la igualdad en todos los sentidos, por la, digamos, adquisición de derechos global, y entonces, ahí estaba globalizado el derecho de la mujer también. Y además, dentro de la clase, asumían los papeles de coordinación tanto chicos como chicas, y los papeles de los equipos eran rotativos: el equipo de limpieza eran chicos y chicas, y limpiaban todos los días. Eso va haciendo una conciencia también, aunque no sea una conciencia expresa ni manifiesta explícitamente, eso crea conciencia de participación (2. ${ }^{a}$ Entrevista, 47-48).

Los problemas con la administración han sido permanentes, así como las luchas de una comunidad para darle continuidad a un proyecto educativo siempre con la amenaza de desmoronarse por los procedimientos administrativos de cambiar el equipo docente implicado en dicho proyecto. Cada año, ante los problemas con la Administración las familias se movilizaban junto al profesorado

5. Se emplea indistintamente, también en la documentación, los términos mixta y coeducación. 
Cada año teníamos una movida que nos deshacía el equipo, todos los años sin fallar, y todos los años como sabíamos que el tema estaba así, en cuanto la más mínima noticia de traslado, se fletaban un par de autocares, los padres y los niños se venían con nosotros y tomábamos la Delegación, pero así, es decir, nos sentábamos de arriba abajo en las escaleras de la Delegación hasta que nos recibían, y cuando nos recibían los padres decían: "no nos vamos de aquí sin nuestros maestros» (1. ${ }^{a}$ Entrevista, 10-11).

Se incorporaba gente que no asumía el proyecto "porque una de las características del Trabenco es que se sigue trabajando más horas de la cuenta, incluso ahora" (1. ${ }^{a}$ Entrevista, 20), y esto creaba ciertas tensiones y desgaste a lo largo de los años por las permanentes explicaciones, discusiones y negociaciones con el profesorado de nueva incorporación.

Distintos son los mecanismos que se utilizaron para dar estabilidad y continuidad al equipo docente al menos durante un año: participar en convocatorias de proyectos de innovación o convertirse en un centro de integración cuando siempre lo había sido, es decir, oficializar acciones que formaban parte del proyecto.

La administración ni comprende ni acepta que el centro lo gestionaran conjuntamente familias y docentes, por ello se nombran los cargos unipersonales solo para cubrir una exigencia administrativa. A Lola le tocó durante algunos años ser jefa de estudios.

... eso lo "chanchulleabamos» siempre claro, porque nosotros teníamos un equipo directivo colegiado y eso no existía de cara a la administración, entonces tenía que figurar un director nominal y se ponía uno, se iba pasando el tema y había que tener un jefe de estudios nominal, también había que tenerlo por allí, y bueno lo íbamos haciendo sobre el papel. Pero en la realidad era un funcionamiento colegiado. La asamblea general era decisoria... (1. ${ }^{a}$ Entrevista, 14).

Trabenco "era la vida" (2. ${ }^{a}$ Entrevista, 70 ), estaba diluido en la vida del barrio, no había muros, ni "reales" ni simbólicos, formaba parte de una cotidianidad construida en común.

Estar en Trabenco le ha supuesto a Lola «toda una vida, claro, son 18 años de mi vida, ha sido más vida que trabajo. Porque yo me estaba haciendo como persona allí también, a la vez que el colegio» (2. ${ }^{\text {a }}$ Entrevista, 33).

Lola inicia su implicación en Trabenco con «la ilusión de poder hacer allí algo de lo que se estaba haciendo en otras partes del mundo con gente que tenía posibilidades y allí no las había...” (1. ${ }^{a}$ Entrevista, 10). Es a través de su participación en un proyecto político de educación, especialmente durante los primeros años, cuando va tomando conciencia sociopolítica, que coincide con sus dudas y cuestionamiento con respecto a las creencias religiosas que en aquel momento tenía

Sí, sí, porque luego ya coincide con los primeros años de Trabenco, en que yo tomo una conciencia política, claro, política y social. [...] Yo diría que fundamentalmente. Los primeros años de Trabenco eran de encuentro con la gente, con gente más concienciada, con gente de izquierdas, los cooperativistas, las asambleas, el 
hablar con los padres de los alumnos, el ver sus realidades. Eran unos años que también el ambiente era así. El ambiente era propicio a eso. En los años 70, al final del franquismo, estaba todo el mundo mucho más concienciado políticamente, ¿no? (2. ${ }^{a}$ Entrevista, 18/19-20).

Este proceso de tomar conciencia fue un proceso de aprendizaje no exento de conflictos con compañeros y compañeras, conflictos que al mismo tiempo iban conformando un ámbito de debate común.

El curso 1990/1991 fue el último de Lola en Trabenco, decidió no continuar

... di la experiencia por cerrada, me pareció que ya tenía que cerrar, tenía que entrar gente joven, y aquello cambiar de algún modo, actualizarse y nosotros ya habíamos cumplido un papel. [...] Quería conocer un centro normal (1. ${ }^{a}$ Entrevista, 1/23)

Solicita un año sabático para realizar un proyecto de investigación, concedido por el Ministerio, para ordenar todo el material, recopilar la información, reflexionar y escribir sobre el trabajo realizado en Trabenco en relación a la educación plástica y la tutoría, y tomarse un año de transición.

Finalizado el año sabático, se incorpora en un centro público de la localidad de Rivas-Vaciamadrid (Madrid). Comienza en el ciclo superior de la educación primaria, permanece durante siete años en este, viéndose forzada a pasar a secundaria al ser licenciada, nivel en el que permanece cinco años. Tiempo que recuerda de manera crítica por la obsesión de contenidos que hay en esta etapa, donde predomina la figura del especialista -profesor-, dejando en un segundo plano la etapa evolutiva del niño/niña, y reivindicando la necesidad del papel de maestro o maestra.

Durante los años en el nivel de primaria trabaja en equipo. Sin embargo, en secundaria son años de trabajo más solitario. Introduce innovaciones en su aula -no sin resistencias por parte de la dirección del centro-, sin lograr contagiar al equipo docente. Considera que no hacía «ni una mínima décima parte que en Trabenco». De su capital pedagógico mantiene durante estos doce años: una estructura espacial en el aula que permite la comunicación y el trabajo cooperativo; la asamblea de aula con el sistema de Freinet de felicito, critico y propongo; la evaluación continua, las «hojas de ida y vuelta que les llamaba, pues yo les escribía alguna cosa [cualquier cuestión personal], ellos me devolvían la contestación...» (2. ㄹ Entrevista, 4); el trabajo interdisciplinar de tutoría-educación plástica-lengua, así como algún encuentro con escritores y escritoras.

Decide jubilarse el año que cumplía 60 años, pero los contactos con Trabenco continúan, ha colaborado con el centro después de su marcha.

\subsubsection{La concientización para la participación en un proyecto de educación alternativo}

Consuelo Uceda inicia sus estudios en 1960 y los concluye tres años más tarde. En el transcurso de estos, con la edad de 15 años, por mediación del sacerdote que le impartía clase, se vinculó, al igual que otras compañeras, a un grupo de Acción 
Católica, que seguía la línea de Pierre Teilhard de Chardin ${ }^{6}$. Dicha vinculación incide posteriormente en su trayectoria docente en tres aspectos claves: adquisición y acumulación de un capital social, que influye en las ofertas de destinos profesionales; el aprendizaje de la metodología de investigación acción relacionada con temas religiosos, que con los años la traslada e incorpora a su práctica educativa; y el valor del trabajo en equipo, que será uno de los principales criterios para valorar posteriormente su experiencia docente en los distintos proyectos educativos en los que ha estado vinculada y el aspecto que más le ha marcado en su trayectoria profesional, tener un grupo o no de trabajo con el que reflexionar, debatir y compartir sus experiencias.

El trabajo en equipo lo inicia en este grupo de Acción Católica, participando en las Ciudades Juveniles, encuentros que se realizaban durante las vacaciones de verano. En estos, el grupo se organizaba en equipos, tenían responsabilidades en cada una de las tareas que realizaban, debatían sin jerarquías temas religiosos. De esta participación destaca el aprendizaje de la responsabilidad y el compromiso, tanto por el trabajo que realizaba como, y especialmente, por la confianza que su familia depositaba en ella, dándole una libertad que en aquella época pocas mujeres adolescentes tenían.

Son años que vive al margen de la política, centrada en sus estudios de Magisterio, en la formación y acción religiosa, en los que la amistad y el tiempo compartido entre iguales era el centro de su vida.

No tenía ni idea de política, la única idea que podía tener era que en mi casa jamás se hablaba de política ni de qué había pasado en la guerra, nada, nada, jamás. Y yo, yo que esto era una dictadura, ni idea. No sé, que no tenía ninguna formación política, nada más que la que ellas ${ }^{7}$ me daban. La verdad es que veía actitudes en ellas que me repateaban muchísimo (1. ${ }^{a}$ Entrevista, 14-15).

Es cuando estudia Pedagogía, de 1965 a 1970, y vive los movimientos estudiantiles contra la dictadura cuando va tomando conciencia de la realidad sociopolítica del país, comienza su militancia y activismo político en el Partido Comunista, formando parte de la célula del PCE del sector de enseñanza cuando reingresa al cuerpo docente, activismo que se ve reducido en el 73, al nacer su primer hijo.

... esos años fueron los de, en fin, el descubrimiento de lo que pasaba en España. Hasta entonces, nada, nada de nada. Y para mí la universidad, más que las clases, fueron todas las vivencias de ambiente estudiantil, de persecución, de ver a los grises entrar a caballo en la facultad. Entonces, para mí fue un aprendizaje muy, muy importante. Los años 68, 69 me pilló en la facultad, conocía a gente del PC y me uní a ellos. Entonces, pensando, en realidad porque la experiencia esta cristiana de base que yo tenía, no estaba tan lejos. Luego se dice «ah, el comunismo, en contra

6. Religioso -de la orden jesuita-, paleontólogo y filósofo francés.

7. Se refiere al curso impartido por la Sección Femenina, obligatorio para aprobar Magisterio. 
de la Iglesia", no. El cristianismo que yo había aprendido y había compartido, se parecía bastante al movimiento comunista. Era el compromiso, lo solidario, de trabajo por los más débiles, estaba bastante cerca y la organización que había, la gente que yo conocía pues me hizo entrar en eso... (1. ${ }^{a}$ Entrevista, 10-11).

Previamente a cursar los estudios universitarios de pedagogía tiene dos experiencias docentes. Una, justo al finalizar los estudios de Magisterio con 17 años, mientras prepara oposiciones, imparte clases en una escuela oficial privada, instalada en un piso del barrio de la Concepción en Madrid, caracterizada por un modelo de enseñanza tradicional. Otra, al año siguiente cuando aprueba las oposiciones, en preescolar, en una escuela segregada rural en Brea del Tajo (Madrid). Resalta de esta experiencia la dificultad de trabajar y atender a un caso específico de un niño, y constata en general la falta de formación recibida en Magisterio, más centrada en contenidos de distintas áreas de conocimiento que en aspectos didácticos, formación básicamente teórica y nada práctica.

En el concurso de traslados le adjudican, para el siguiente curso escolar, 19651966, un pequeño pueblo de la sierra de Madrid, Navarredonda. Tras visitar la localidad, decidió no ocupar la plaza, ya que se veía sola y aislada en aquel lugar, y optó por seguir estudiando para tener una mayor formación pedagógica. Comenzó Pedagogía y a interesarse por la innovación educativa, buscando respuestas a cuestiones de la realidad del aula tras la experiencia en Brea. En esta búsqueda va uniendo la educación con lo político y lo social.

... por otro lado, entonces también tenía esta otra experiencia de trabajo con gente de solidaridad, de relación... Eso iba por ahí. Y en la Universidad empecé a ver que había otras cosas: política, tal, la idea de la libertad, de que estábamos en una dictadura, que, para mí, antes me daba igual, no sabía... Entonces, digamos que confluían las dos cosas, ¿̇no? Y la visión política me dio más una idea más de democracia, de libertad, de derechos a la educación de todo el mundo, de esa justicia social... Y por el otro lado estaba, digamos, la vía más pedagógica, más didáctica, más liberadora... (2. ${ }^{a}$ Entrevista, 52$)$.

Durante los dos últimos años de estudios asiste a las Escuelas de Verano de Rosa Sensat y conoce los Movimientos de Renovación Pedagógica. Son años en los que se sitúa en una línea pedagógica política crítica, en una vinculación teóricopráctica, optando por un modelo de enseñanza innovador, que la lleva a un permanente cambio y búsqueda, "quizás no estar nunca suficientemente satisfecha" (2. ${ }^{a}$ Entrevista, 43), donde la incertidumbre es permanente porque «tienes que arriesgar para hacer eso» (2. ${ }^{a}$ Entrevista, 43) y asumir una concepción de maestra no ligada al modelo maternal característico de la época, sino con un papel de potenciar el crecimiento de niños y niñas

Yo soy trabajadora de la enseñanza en un servicio público, siempre he trabajado en la escuela pública y para la escuela pública... [...] Yo tengo una idea de que hay que educar para hacer ciudadanos libres... libres, solidarios, democráticos, laicos... esas cosas, ¿no? Y, por tanto, todos los instrumentos y todas las metodologías, todo 
lo que vaya en esa dirección de hacer ciudadanos libres, críticos, me parece que es como hay que trabajar... (2. ${ }^{a}$ Entrevista, $\left.70-71\right)$.

Finalizados sus estudios de Pedagogía, le llega la información, a través de una persona conocida del grupo de Acción Católica, que hay una oferta de plazas en la Fundación Hogar del Empleado ${ }^{8}$, la cual tenía varias Fundaciones dedicadas a la educación, con un acuerdo con el Ministerio de Educación: ponía a disposición los locales y el ideario pedagógico y el Ministerio al profesorado funcionario. Se presenta a la entrevista previa para la selección, es seleccionada y comienza a trabajar en la Fundación, en Bachiller por ser licenciada, en el curso 1970-1971. "Pero lo mío era la escuela", y por ello decide cambiar al siguiente curso académico hasta 1980 al nivel de educación primaria. Toma dicha decisión porque considera que en secundaria hay un afán y práctica de especialización, una obsesión por los contenidos y la calificación, sin trabajo de coordinación ni de equipo, donde se pierde el sentido de ser maestro/maestra. Son años de vinculación teórico-práctica, en un proyecto pedagógico centrado en el contexto del aula, partiendo de la participación del alumnado. Participación definida por el trabajo en grupo, la cooperación, el diálogo, la discusión y el debate, la formulación de propuestas de trabajo y la autonomía.

La asamblea de aula configura el trabajo de esta. Era también el espacio de felicitar, criticar y proponer, siguiendo a Freinet, donde se debatían y consensuaban las normas de clase y las del patio. Esto requería romper la estructura jerárquica del aula, tanto en lo referente a espacios como a tiempos. Estos se configuraban en función de lo que estaba ocurriendo en cada momento, en función de la propuesta de trabajo que se estaba realizando y no por la entrada de especialistas. El aula se organizaba por rincones o zonas, que reflejaban el resultado de los proyectos de investigación que realizaban, tras la utilización de distintos recursos y fuentes de información, contando con el libro de texto como apoyo.

Tras diez años de trabajo docente en la Fundación decide cambiar y concursar a una escuela rural, guiada por su máxima (no estar en el mismo centro más de 10 años para evitar la acomodación) y hacer real su imaginario: "ser maestra de pueblo», configurado a partir de la figura de su madre, en el que une la procedencia de esta y la decisión que tuvo de que ella fuera maestra.

Le adjudican una plaza en un pequeño pueblo, que no llega a ocupar, ya que una amiga le informa de un proyecto educativo que se está llevando a cabo en el centro Príncipe de Asturias, perteneciente entonces a la Universidad Autónoma de Madrid, con el mismo sistema de concierto con el Ministerio que la Fundación, y la anima a visitarlo. La admitieron tras la realización de las pruebas para la selección de docentes, y considera «esa etapa, que fueron sólo cuatro años, pero para mí, como toda la vida» (1. ${ }^{\text {a }}$ Entrevista, 23).

8. FUHEM tiene sus orígenes en 1949. Fundación que aborda los problemas de la ciudad de Madrid en los años cincuenta y sesenta (vivienda digna, barrios equipados con diferentes servicios, etc.). 
Toda la vida porque le permitió un mayor desarrollo profesional. Podía hacer todo lo que había venido desarrollando en el centro de la Fundación y además realizar otras prácticas que iban en la línea pedagógica que ella asumía y defendía. Son años de trabajo en equipo, de formación conjunta en las escuelas de Verano de Acción Educativa, de coordinación para la elaboración de planes de trabajo y de materiales propios -ya que no utilizaban libros de texto-, de seguir una metodología globalizada por medio de proyectos de investigación que realizaban en grupos, de practicar la asamblea como núcleo y apertura a múltiples y diversos aprendizajes -hablar en público, dialogar, organizarse, responsabilizarse, etc.que continuaban con los diferentes compromisos adquiridos, estando presente la pedagogía del cuidado, de la ocupación y preocupación por el otro. Al mismo tiempo, se mantiene una relación con el medio para un conocimiento del entorno, mediante salidas, campamentos, etc. La evaluación no consistía solo en calificar, sino que realizaban informes de evaluación que entregaban a las familias mediante entrevistas individuales. Estas estaban más presentes en el centro, había un cierto nivel de participación en lo reglamentado. También el alumnado realizaba prácticas de autoevaluación (Uceda, 2005).

En 1984 le ofrecen formar parte del equipo de asesoramiento de la Comunidad de Madrid, cuando se crea la Dirección General de Educación, en programas educativos de apoyo a la escuela en distintas temáticas: salud, consumo, medio ambiente, etc. Durante tres años desarrolla proyectos para promover los servicios que tenía la Comunidad vinculándolos a la escuela.

En 1987 le proponen desde el Ministerio de Educación formar parte del equipo que se encargaría del Diseño Curricular Base y de los programas de experimentación de la LOGSE. Propuesta que acepta "porque me parecía una oportunidad de transformar la escuela, de ver si las escuelas como las que yo conocía, de innovación y tal, se podían generalizar a través de lo que se iba a hacer..." (1. ${ }^{a}$ Entrevista, 30). Coordina un equipo como jefa de servicio en el Ministerio para el diseño curricular de la etapa de Primaria, que elabora la documentación de la misma que forma parte de las Cajas Rojas. Años de trabajo intenso, de trabajo en equipo, de debate y reflexión colectiva, en los que adquiere nuevos aprendizajes, profundiza en el constructivismo, va teorizando su práctica docente, adquiriendo un mayor desarrollo y profundización teórica.

En el año 92 da por finalizada esta etapa de trabajo en el Ministerio, "se había puesto en marcha en los primeros cursos y otra vez, yo, no me quería quedar allí. Yo fui a la administración para trabajar, pero no me quería quedar allí. [...] pensé que había hecho todo lo que se podía hacer ahí. Y luego yo ya quería volver a la escuela a ver si era verdad que se podía hacer» (1. ${ }^{a}$ Entrevista, 32 ).

Participa en el concurso de traslados con la idea de ir a una escuela rural, que seguía en su imaginario, y le adjudican un pueblo en la sierra de Madrid, Cercedilla. No es una escuela de esta índole, sino graduada, "una escuela normal, de todo, con profes normales, con libros de texto, con ninguna experiencia innovadora..." (1. ${ }^{a}$ Entrevista, 32-33). Inicia esta experiencia en primero de primaria, la primera 
vez que impartía clase en este curso, incorporando en su aula lo que había venido caracterizando su trabajo como docente: las asambleas, la investigación, las salidas al medio, la construcción de un conocimiento compartido a través del diálogo y la puesta en común, grupos de trabajo y responsabilidades, distintas fuentes de información, el texto libre, la relación con el medio, etc. Realiza un trabajo básicamente en solitario, sin apenas coordinación.

Nuevamente, a través de una persona conocida le llega la información de la posibilidad de ir a un centro, al CEIP La Navata ${ }^{9}$ en Galapagar-Madrid, que viene desarrollando un proyecto educativo, "Hacia una escuela para todos», elaborado en 1994 por un equipo docente respondiendo a la convocatoria del MEC «77 medidas para la calidad de la enseñanza", siguiendo las orientaciones de la LOGSE (Uceda, 2006). Convocatoria que ofrecía la posibilidad de que el equipo que lo presentaba lo desarrollase en un centro de nueva creación. Se inicia el proyecto en las instalaciones de la escuela unitaria existente y otras prefabricadas hasta 1997, año en el que se construye un nuevo edificio.

Cansada de una jefatura de estudios burocrática y poco pedagógica, tras visitar el colegio de La Navata, hablar con el equipo docente y conocer el proyecto, decide incorporarse a dicho equipo en el curso escolar 1999-2000.

Los principios educativos del proyecto son la atención a la diversidad con la participación e inclusión de las distintas culturas; la educación ambiental, con el objetivo de conocer, analizar y reflexionar sobre el medio ambiente para su cuidado y mejora; la comunicación a través del desarrollo de diferentes lenguajes -oral, escrito, musical, plástico, corporal y audiovisual-; y la participación del alumnado y de la comunidad educativa.

En relación a la participación de las familias, el centro está abierto a estas, diariamente se dispone de un tiempo al iniciar la mañana para entrar en el aula y hablar con la tutora o tutor. Cualquier aspecto puede ser debatido, se comparte la tarea de enseñar (Feito, 2005, 2006), discutiéndose la programación y los planes de trabajo con ellas, así como los proyectos de trabajo comunes en el centro -se realiza uno por trimestre-. Son conocedoras de las actividades del aula a través del Diario de a bordo, que diariamente escribe un niño o una niña, sistematizando qué se ha hecho a lo largo del día, qué ha sucedido, etc. Las familias participan de forma activa en los talleres que se realizan por la tarde por ciclos. Existe, además de los cauces recogidos por la normativa vigente -Consejo Escolar y AMPA-, una asamblea de madres y padres, con representantes de los tres ciclos que se reúne una o dos veces al trimestre con la directora para tratar distintos aspectos del centro. Este cauce de participación también existe con el alumnado a través de representantes de cada una de las clases para abordar temas que derivan de estas o aspectos más relacionados con los espacios comunes.

9. http://cp.lanavata.galapagar.educa.madrid.org/. 
El alumnado tiene un papel activo en la construcción de sus aprendizajes. El trabajo del aula presta atención al desarrollo emocional y afectivo de niños y niñas (Uceda, 2006) y parte del conocimiento que poseen para potenciar el aprendizaje significativo y lo que desean saber y sus intereses, que se manifiestan en la asamblea de aula, saliendo también de estas propuestas para los talleres. Para ello el trabajo en grupo es básico, realizándose distintos tipos de agrupamientos con distintas funciones y responsabilidades respecto a la biblioteca, el material, pasar lista, etc. Los contenidos curriculares se conectan con la realidad (Feito, 2006).

El trabajo del aula no se sustenta en el libro de texto, este material no se utiliza, los materiales son elaborados por el profesorado y se utilizan diferentes fuentes de información. El aula se organiza por zonas o rincones, en la que hay distintas propuestas de trabajo, con tiempos flexibles, pudiendo el alumnado decidir, una vez concluida la propuesta mínima, en qué zona invertir su tiempo. Dichas zonas se conectan con la destinada al proyecto de investigación. Por ello los tiempos no están fraccionados, son globalizados (Uceda, 2006). Formaba parte de este proceso la elaboración de El libro de las cosas importantes, "que era lo que todos íbamos a aprender [...] eso era lo que todos tenían que saber, que era lo básico del tema que habíamos trabajado. Y luego cada grupo y cada uno sabía 15 veces más de lo que le había tocado... pero ofrecía a los demás la información básica...» (2. Entrevista, 27), en correspondencia con la idea de enseñanza comprensiva "lo mínimo para todos y lo máximo para cada uno» (1. ${ }^{a}$ Entrevista, 28).

La organización de los distintos recursos educativos -tiempos, espacios, materiales, agrupamientos- responde a una línea de trabajo que tiene como finalidad potenciar la comunicación, la participación, la autonomía, la responsabilidad, la cooperación, el valor de la diversidad, de lo público, lo común. Con esta idea la cooperativa de padres y madres proporciona y gestiona el material.

La evaluación es continua y formativa, realizándose en distintos tiempos y espacios: durante el proceso de los proyectos de trabajo (recogida, selección, elaboración y puesta en común de la información), con una escucha activa permanente en las asambleas a la oralidad del alumnado en relación a exposiciones e intervenciones, y con la revisión de los trabajos escritos referentes a las distintas zonas del aula, donde el error es el punto de partida para seguir avanzando y no para calificar o tachar, sino para realizar anotaciones a modo de guía, sugerencia o revisión. También forma parte de esta evaluación la coevaluación entre iguales en el momento de hacer públicos sus aprendizajes y la autoevaluación al finalizar un proyecto de trabajo o un trimestre, en la que cada niño y niña tiene que reflexionar sobre lo que ha aprendido, qué le ha gustado y qué no, qué aspectos debe mejorar, así como realizar una evaluación de la maestra. Toda esta información es integrada en un informe que se le entrega a las familias, ya que en La Navata no hay notas.

Comenzar a trabajar en La Navata le posibilitó poner en práctica de forma colectiva el conocimiento acumulado en su trayectoria profesional. Sin embargo, el equipo de trabajo se dividió en el momento en que desaparecieron las comisiones de servicio y la administración no facilitó que las nuevas incorporaciones fueran 
conociendo y asumiendo un proyecto de base, posibilitando dar continuidad a un proyecto de manera no conflictiva por profesionales que tienen otra concepción de la enseñanza y del aprendizaje.

Existiendo esta situación, tuvo que asumir la dirección del centro durante tres años por nombramiento y renovación del mismo por parte de la inspección, informada y a propuesta de las familias y del equipo docente cuando la anterior directora se jubiló y nadie quería ocupar el cargo.

Como directora del centro se marcó como objetivo integrar posturas, manteniendo las bases del proyecto educativo, pero, "de alguna manera, había que ir flexibilizando el tema, sin perder lo esencial, pero flexibilizando para que todo el mundo se sintiera implicado. Pero con demasiada frecuencia ocurría "las del proyecto" y "las nuevas" y eso creaba una división" (2. ${ }^{a}$ Entrevista, 4). Por esta razón nombró como jefa de estudios a una maestra que se incorporó al proyecto al constituirse La Navata con la intención de incorporar en el equipo directivo al sector crítico y con resistencias al proyecto, como estrategia que podía favorecer a su desarrollo estando dentro de los órganos unipersonales.

Puso en marcha otras estrategias ante las resistencias continuas de parte del profesorado, y con el objetivo de que maestros y maestras de nueva incorporación se sintieran integrados en la dinámica de trabajo del centro y acompañados en prácticas educativas distintas a las hegemónicas, al mismo tiempo que perseguía dar coherencia a las acciones de un equipo docente. Resistencias debido al tiempo que implica el ejercicio de la autonomía, la elaboración de materiales, la evaluación del alumnado con informes, etc. Una estrategia fue que en cada curso y ciclo debía haber una persona con trayectoria acumulada en el proyecto de centro y una persona sin dicha experiencia, evitando así que las personas recién llegadas se retroalimentaran en las dificultades que entraña trabajar de otra forma y no en las posibilidades. Dicha decisión no estuvo al margen de cierta queja o polémica por parte del sector que asumía el proyecto por considerar que era una medida que le limitaba a cierto estancamiento. Este mismo malestar se producía cada año, ante las nuevas incorporaciones y la inestabilidad del equipo docente, cuando se realizaban en septiembre sesiones de formación. Estas suponían anualmente explicar el proyecto, convencer de las potencialidades del mismo, era estar todos los años en el mismo punto, aflorando el sentimiento de estancamiento, de un avance lento o de un continuo freno.

Otra estrategia, con el objetivo de integrar, fue decidir que el primer proyecto de trabajo del año escolar fuera común, "venimos de vacaciones», con fin de elaborar materiales comunes que pudieran guiar a las maestras y maestros recién llegados. Dicha estrategia formaba parte de otra más amplia: elaborar materiales y generar una base documental para guiar, sugerir y utilizar. Sin embargo, había resistencias ya no solo en elaborarlos, sino también en compartirlos, por miedo a ser juzgados y juzgadas.

Al mismo tiempo, desde la dirección realizó un trabajo encaminado a cambiar concepciones y prácticas arraigadas que convertían la participación real del 
alumnado en pseudoparticipación. Asimismo, no aceptó retomar el debate de jornada continua-jornada partida, que en su momento se decidió partida por mayoría del profesorado y de las familias, pero que generó «un enfrentamiento brutal», disfrazando las razones e intereses personales de razones pedagógicas. Es partidaria de centros abiertos con profesionales diversos, no entendiendo la jornada partida como la extensión de la jornada docente ni como una prolongación de las actividades de la mañana, sino un tiempo para posibilitar otras experiencias al alumnado.

En 2007 decide jubilarse, incidiendo en esta decisión dos factores: uno, estar cansada de la dirección, un espacio conflictivo y poco deseado; y otro, ser consciente de un cambio que se produce en su concepción y apreciación de los niños y niñas, cobrando relevancia, según Consuelo, la diferencia generacional.

Deja la escuela, pero no la educación, sigue vinculada al Movimiento de Renovación Pedagógica de Acción Educativa con un grupo de trabajo que desarrolla el proyecto "La ciudad de los niños", en la línea de Tonucci, con un convenio con el Ayuntamiento de Madrid con el Programa "Madrid a pie», con el objetivo de que el Ayuntamiento promueva la participación de los niños y niñas en la ciudad.

\section{LAS ALTERNATIVAS PEDAGÓGICAS: «INTERFASE» PARA LA CONSTRUCCIÓN DE OTRA EDUCACIÓN}

Alternativas pedagógicas ${ }^{10}$ democráticas como Trabenco y La Navata, ejemplificadas a través de las prácticas de Lola Pedrosa y Consuelo Uceda, como hemos visto, que, grosso modo, construyen proyectos educativos colectivos, sustentados en la participación activa de docentes, alumnado y familias, vinculados a lo político y a lo social a través del ejercicio de la democracia tanto en la gestión de las escuelas como en el desarrollo del currículum por medio de metodologías activas, que permiten construir y reconstruir el conocimiento de manera interdisciplinar y globalizada desde una perspectiva crítica, empleando los recursos diversos existentes, entre ellos el entorno, y de elaboración propia para una educación integral y conectada con la realidad de sus comunidades para el desarrollo de estas y para promover el aprendizaje relevante y autónomo, pueden constituir una "interfase» (Adamovsky, 2011), una transición a modelos educativos radicalmente diferentes a los ya existentes. Modelos que nos encontramos en la propuesta de desescolarización de Ivan Illich (1971) o en la Educación Popular de Paulo Freire. Ejemplos de estos los hallamos en distintos proyectos latinoamericanos que constituyen la Otra Educación, tales como: el Movimiento Sin Tierra en Brasil, los Bachilleratos Populares en Argentina, las Escuelas Zapatistas en México, la Universidad de la Tierra en México o las Escuelas Libres en Chile.

10. Se pueden consultar otras en el Centro de Documentación de las Alternativas Pedagógicas [CEDAP]. http://centrodedocumentacioncritica.org/areas/educacion/archivo-cedap/. 
Trabenco y La Navata son proyectos educativos viables, pueden ser modelos de referencia y, además, pueden constituir una interfase a un cambio educativo y social que no sea visto como una amenaza, sino como la posibilidad de ir reemplazando el modelo hegemónico de educación extendiéndose y ampliándose en su concepción y práctica democrática para la formación de una ciudadanía crítica y activa. Para dicho cambio se requiere establecer conexiones con la educación no formal, con otros centros educativos, con las universidades, los partidos políticos, los sindicatos, las asociaciones, otras instituciones, etc. Es decir, establecer una red, un desarrollo rizomático, que posibilite el cambio hacia la Otra educación. De ahí que sea necesario que los proyectos educativos que constituyen alternativas pedagógicas democráticas realicen no solo una doble apertura: hacia dentro -posibilitando que la comunidad escolar participe y utilizando su capital cultural- y hacia fuera, utilizando los recursos de los barrios-ciudades y participando de sus actividades socioculturales. Debe procurar que la educación se vincule con la experiencia, considerando esta como la totalidad de relaciones de las personas con su ambiente (Dewey, 2004). Esta conexión implica tomar en consideración que niños, niñas, adolescentes, jóvenes y gente adulta forman parte de una pluralidad de grupos, instituciones, asociaciones, etc., en el barrio, en la comunidad. Considerar esta experiencia implica que el barrio es el punto de partida. Se requiere proyectos educativos de barrio, de comunidad, elaborados a través de la construcción y desarrollo de una democracia comunitaria, creada sobre condiciones reales para establecer un espacio común, que permitirá "construir colectivamente el empoderamiento comunitario, endógeno y participativo" (Calle, 2011, 37). Este empoderamiento posibilitará la reconstrucción del espacio público, la base social desde la que será posible la concienciación de la realidad y la acción crítica sobre ella, es decir, educarnos en ciudadanía.

Al mismo tiempo, el proyecto educativo de barrio pensado para su transformación podría constituir una «interfase» autónoma (Adamovsky, 2011) para un cambio social de lo instituido. Es decir, permitiría articular lo educativo con lo social para posteriormente articularlo con lo político.

Trabenco y La Navata, como alternativas pedagógicas, podrían constituir una interfase para la construcción de proyectos de comunidades educadoras o de barrio, que, además, posibilitarían que la educación estuviera diluida en la vida de las personas.

\section{REFERENCIAS BIBLIOGRÁFICAS}

Adamovsky, E. (2011) Problemas de la política autónoma, en Calle A. (ed.) Democracia radical. Barcelona, Icaria, 99-128.

Calle, A. (2011) Aproximaciones a la democracia radica, en Calle A. (ed.) Democracia radical. Barcelona, Icaria, 15-51.

Centro de Educación Infantil y Primaria Trabenco (2007) Proyecto Educativo. Multicopiado. 
Cooperativa de Viviendas Trabenco (1980) Trabenco la realidad de una idea. Trabenco a los 10 años. Madrid, Ayuntamiento de Leganés.

Dewey, J. (1998) Democracia y educación. Madrid, Morata.

Dewey, J. (2004) Experiencia y educación. Madrid, Biblioteca Nueva.

FeITO, R. (2005) Otra escuela es posible: El ejemplo del CEIP "La Navata". Consultado el 31 de mayo de 2017. http://www.ampacolegioalhambra.org/article. php3?id_article=34\&var_recherche=Feito.

Feito, R. (2006) El ceIP La Navata. Una escuela democrática, en FeITo, R. Otra escuela es posible. Madrid, Siglo XxI, 117-146.

García Gómez, T. (2004) El turno de la educación dominada. El Nudo de la Red, (3-4), 3-7.

GARCía GÓMEZ, T. (2011) Rompiendo muros. La educación democrática: proyecto comunal de ciudadanía. Revista Electrónica Interuniversitaria de Formación del Profesorado, 14 (2), 57-69.

Illich, I. (1971) La sociedad desescolarizada. Barcelona, Barral Editores.

PujADAS, J. J. (1992) El método biográfico: El uso de las historias de vida en ciencias sociales. Madrid, CIS.

PuMARES, L. (2001) Estudio de los factores que posibilitan la continuidad de un proyecto curricular global en un medio social determinado: Trabenco, 25 años de innovación educativa. Tesis de doctorado. Madrid, Universidad de Madrid (España).

Uceda, C. (2005) Maestra de por vida, en Imbernón, F. (coord.) Vivencias de maestros y maestras: compartir desde la práctica educativa. Barcelona, Graó, 77-84.

UCEDA, C. (2006) Una escuela para todos y entre todos, en Ministerio de EduCACión y CIENCIA El protagonismo del profesorado: experiencias de aula y propuestas para su formación. Madrid, Consejo Escolar del Estado, 147-152. 